\title{
LA JOVEN NARRATIVA ARGENTINA DE LOS '90': ¿ NUEVA O NOVEDAD?
}

\author{
Edgardo H. Berg*
}

"Hoy. la censura es el mercado"

(George Steiner)

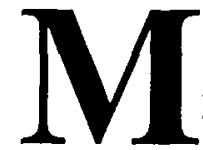

e propongo a partir de estas notas críticas, trazar un mapa de lectura y un primer diagrama cognitivo sobre la producción narrativa de los escritores argentinos que hoy rondanentre los 30 y 45 años, examinando algunos ejes problemáticos relevantes.

La competencia entre algunas casas editoriales en alianza con algunos suplementos culturales capitalinos ha desatado un peculiar enfrentamiento por la legitimidad, el prestigio cultural y la conquista de espacios en la

* Universidad Nacional de Mar del Plata. CELEHIS.

1 El corpus narrativo observado es el siguiente: BIZZIO, Sergio, Infierno Albino (1991); CAPARRÓS, Martín. Ansay o los Informmios de La Gloria (1984); La Noche Anteior (1990) y. El Tercer Cuerpo (1990); CHEFJEC, Sergio. Moral (1990) u El Aire (1992); FEILING. Carlos Eduardo. El Agua Electrizada (1992) y Un Poeta Nacional (1993); FORN. Juan. Nadar de Noche (1991); FRESÁN. Rodrigo. Historia Argentina (1991) y Vida de santos (1993); GUEBEL. Daniel. La Perla del Emperador (1990) y Los Elementales (1992); LANATA. Jorge. Polaroids (1992): PAULS, Alan. El Pudor del Pornografo (1984) y El Coloquio (1990); SACCOMANNO. Guilherme. Bajo Bandeira (1991) y Animales Domesticos (1994). 
llamada "industria cultural". ${ }^{2}$ A su vez, equipos de editores, periodistas y críticos de ciertas revistas culturales desarrollan una atención especial por los libros de autores amigos o del mismo grupo de pertenencia intelectual; por la difusión de los productos culturales puestos en circulación por las casas editoriales a las que se hallan vinculados contractualmente o por la difusión de las preferencias literarias de los mismos escritores. También, subsidios de fundaciones culturales y becas internacionales apoyan y financian a los "nuevos" escritores. ${ }^{3}$

En una sociedad del espectáculo, dominada por la ratio coyuntural y el auge del acontecimiento, el prestigio se calibra muchas veces, por sus efectos: por el éxito en el mercado o por la presencia en los principales medios de divulgación masiva. Los medios y el mercado para estos jóvenes narradores, se convierten en los escenarios privilegiados de la polémica y la legitimación cultural. ${ }^{4}$ Estas nuevas "tribus" o microsociedades de "autobombo" han desplazado la política a las relaciones que mantienen con el mercado, a las tácticas de ingreso en la máquina omnívora de la industria cultural o a la colaboración en los suplementos culturales hoy en disputa. Lucha de intereses a la que no son ajenos los editores y asesores editoriales, los llamados "periodistas culturales", jóvenes críticos o mediadores de lectura y las instituciones del saber literario Universidades, escuelas y otras " academias". 5 Asimismo, estas " sociedades de

2 Me refiero principalmente a la Editorial Sudamericana (colección Narrativas Argentinas) Planeta (colección Biblioteca del Sur) y Emecé (colección Escritores Argentinos). y otras menores como Juan Genovese Editor o Beatriz Viterbo (colección Ficciones). Las dos primeras editorialles - Sudamericana y Planeta-, se hallan asociadas estratégicamente a dos de los principales suplementos literarios capitalinos, respectivamente al Suplemento Cultura $Y$ Nacion del diario Clarin, bajo la dirección de Marcelo Pichón Riviere y Primer Plano de Pagina/l2 que comienza a publicarse en 1991, bajo la dirección de Tomás Eloy Martínez.

3 Tomemos en consideración algunos ejemplos. Luis Chitarroni como asesor de la editorial Sudamericana, crítico del suplemento literario Primer Plano de Pagina/2 y divulgador de algunos escritores amigos, integrantes de la ex revista de critica de libros Babel (1988-1990) y del ex grupo autodenominado "Shangai" (Caparrós, Guebel, Pauls entre otros). Juan Forn, escritor perteneciente al otro grupo literario en disputa y editor de la Editorial Planeta. o su amigo. cl escritor Rodrigo Fresán. columnista de Primer Plano y del Suplemento Joven de Pagina/ 2. También. me reliero entre otras, a las fundaciones culturales pertenecientes a algunos de los principales grupos económicos del pais. Como. por cjemplo, la fundación Antorchas del grupo económico Bunge Born. la fundación del Banco Patricios de Buenos Aires o la regenteada por la prominente empresaria del cemento de Loma Negra. Provincia de Buenos Aires, Amalita Lacroze de Fortabat.

4 Cuando estaba revisando estas mínimas líneas, hallé un interesante análisis sobre la cuestión de la incidencia del mercado, tanto en la configuración artística como en el destino de la circulación social de las producciones estéticas en la actualidad. Para una mejor comprensión del fenómeno cfr. SARLO, Beatríz (1994, p. 27-31).

5 También las preferencias ingresan a la Universidad. Guebel. Pauls y Feiling son invitados en 1991, a debatir con los alumnos de la cátedra de Literatura Argentina de la Universidad Nacional de Buenos Aires. 
autobombo" muchas veces, son artífices privilegiadas del diseño y de la configuración de los espacios del debate cultural en la coyuntura e inciden deliberadamente en la división del mercado de bienes simbólicos. ${ }^{6}$ En este sentido, los debates y polémicas que recorren los encuentros de escritores, organizados por fundaciones culturales o por las casas editoriales dominantes en la actualidad y difundidas por el llamado " periodismo cultural" dan cuenta del estado actual de la relación de los escritores con el mercado, la industria cultural y los virtuales receptores. En muchos casos, los debates literarios tienen más que ver con aquello que Roland Barthes denominó como goce histérico: una forma de teatralización, de show de réplicas y agresiones, de doxas y contradoxas para la consagración cultural o el éxito en el mercado.

Retomo. La solidaridad e interacción dinámica de los diversos agentes del campo cultural, la relación entre escritores, críticos y periodistas culturales, organiza y construye los nuevos grupos o "tribus" literarias. Mejor aún, de "novedades" que el mercado necesita para el consumo. En este sentido, las poéticas narrativas actuales muchas veces, se definen por su relación con el mercado; o mejor aún, el mercado diseña una cartografía de opciones y enfrentamientos. Pero también, las prácticas literarias son formas de lucha. políticas de legitimación dentro del campo cultural.

Sabemos con Pierre Bourdieu que desde que el editor reemplazó en el siglo XVIII al mecenas, la autonomia del arte es siempre relativa a las demandas del mercado. Los escritores son colocados a partir de la Modernidad en situación de competencia y la antigua diferencia entre valor literario y valor mercantil se hace cada vez más estrecha. ${ }^{8}$

Entonces $¿$ nuevos en qué sentido? ¿ novedad para la capilla de editores. asesores editoriales y amigos? ¿ Nuevas poéticas o poéticas del efecto?. Nuevo o novedad, en el sentido que le da a esta palabra el Marketing, se liga a la etiqueta que el mercado ofrece a los virtuales consumidores. Pero, si la categoria de lo nuevo se asocia a aquellas producciones estéticas que producen un verdadero corte y ruptura con la tradición literaria, a "lo historicamente necesario". me temo que la categoria cae por deflación o por primeriza.

6 Cfr. Levin Shücking (1960: p. 44-46). También a modo de ejemplo. es interesante observar los debates y polémicas entabladas entre la anterior promoción de escritores. entre otros Abelardo Castillo, Ricardo Piglia. Luis Guzmán. Juan Carlos Martini. Eduardo Belgrano Rawson. Ana Maria Shua. Héctor Libertella y algunos de los representantes de la llamada "joven" narrativa. Martin Caparrós. Matilde Sanclez. Sergio Bizzio. Daniel Guebel y Sylvia Iparraguirre en la reunión de escritores desarrollada en la ciudad de Pinamar y reproducida en la edición a cargo de (jabricla Saidón (1993).

7 Crf. BARTHES. Roland (1987, p. 135-139).

8 Ver BOUDIEU. Pierre (1971. p. 135-182). 
" La literatura es un ejército en retirada que libra batallas de hostigamiento con la cultura de masas"

(Ricardo Piglia)

¿ Cómo producir una ex-tradición, una fuga sobre el canon dominante de los medios masivos? Creo que esta pregunta atraviesa y condensa uno de los actuales ejes problemáticos del estado actual del debate literario. Sobre nuestro umbral epocal, la literatura y en especial, la narración literaria debe competir contra las ofertas dominantes y hegemónicas de la era de los medios electrónicos de comunicación. Competir con esa "selva de signos" provenientes de diferentes imaginarios y con las nuevas formas de lectura que generan el cine, los seriales de la televisión, el periodismo, el video clip, los recitales de rock y la llamada cultura del zapping.'

Peter Bürger ha trazado la historia de ese gran relato utópico trastocado en fracaso gue constituyó el impulso homicida de la vanguardia europea de los años ' 20 '. "Sabemos que el "asalto" contra las instituciones del arte, el intento surrealista y de los llamados ismos- de liquidación o disolución del arte como una praxis separada de la praxis vital, ha sido cristalizado: y cualquiera de nosotros, puede hoy observar como una obra de vanguardia se ha convertido en el mejor de los casos, en una pieza de museo o en un objeto de decoración de alguna oficina de la gran ciudad. La " tradición" de vanguardia o eso que llamamos posvanguardia o neovanguardia por hábito o a falta de otro término. es la absorción de las intenciones extrartisticas de la vanguardia histórica a fines autónomos. Se trata en definitiva, desde otro contexto epocal y comunicacional

? Nicolás Rosa aporta algunas reflexiones sobre estos nuevos modos de lectura. Sobre la cultura del zapping nos dice: " Frente a la consola y el microprocesador. frente al televisor computarizado. aparece una verdadera tiesta. un shopping cultural, donde se le ofrecen al lector nuevos recorridos. itinerarios inquictantes para que transite atraves del control remoto. una verdadera hecatombe de fragmentos. una lógica del disloque. un texto trozado de piezas provenientes de imaginarios diferentes. una descomposición de géneros: el zapping como lectura. como una verdadera lectura delincuente. Hoy se lee poco pero se lee más voraz-mente. más encarnizadamente”. Cfr. Nicolás Rosa (1992: 35). Habria que estudiar por ejemplo. las relaciones entre la sintaxis que produce la llamada cultura del zapping y la sintaxis narrativa presente en los textos de Rodrigo Fresán.

10 Ctr. el excelente libro sobre la vanguardia de Peter Bürger (1987). Asimismo en un reciente artículo. el autor retoma ciertas reflexiones sobre la crisis de las llamadas vanguardias históricos pero en el contexto del actual debate de la Modernidad/ Posmodernidad. Ver Peter Bürger (1993. 40-48). 
-la instancia de la aparición y el desarrollo de los medios masivos es decisiva-, de la continuación de los movimientos de vanguardia por otros medios. La tradición de la experimentación y la ruptura con el canon hegemónico, la destrucción del concepto tradicional de obra orgánica o de la "redondez" del relato, donde el todo debia concordar con las partes, son algunas de las instancias que desde la defensa de la autarquia y de los mundos posibles del arte, retoma la neovanguardia. Fase artistica que ha sido analizada desde dos ángulos bien diferenciados por Theodor W. Adorno y Walter Benjamin. " Diremos simplificando la cuestión, desde una perspectiva que atiende a los aspectos construtivos y de producción de la obra de arte en el primer caso; y en el segundo, atendiendo a los nuevos modos de recepción de la "obra" - y coloco entre comillas la categoria de obra porque la teoria benjaminiana tiende a su disolución- en el contexto del desarrollo de los medios masivos de comunicación, " medios de reproducción mecánica" diria Walter Benjamín.

Ahora bien, me gustaría destacar dos inflexiones. En primer lugar, lo que me parece unos de los aportes decisivos de la teoría benjaminiana en la resemantización del objeto-arte. Benjam in a diferencia de Adorno pone el acento sobre la nueva recepción que surge del nuevo contexto de producción: pasaje de una recepción propia del rito del culto, de la lejania y inaccesibilidad del arte a la recepción masiva. Vinculada a la pérdida del aura o del contexto aurático de la obra de arte en la época clásica, con la aparición del fenómeno de reproducción mecánica. A partir de este nuevo contexto se articula la disolución de la obra de arte clásica, donde se confunden o se hacen intercambiables el original y la copia, asi como surgen nuevas políticas en el arte por el uso del montaje.

Conviene agregar a este mínimo planteo, la asincronía o mejor, cierto desajuste que supone incorporar la tradición de la vanguardia nacional. Como bien, señala Beatriz Sarlo, lo nuevo para los martinfierristas " es una relectura de la tradición": cruce de la textualidad vanguardista con el criollismo, Marinetti y Gómez de la Serna junto a Evaristo Carriego. ${ }^{12}$ A su vez, tanto Macedonio Fernández como Jorge Luis Borges lejos de acordar con el proyecto extremo del surrealismo, de llevar al arte hasta los extremos mismos de la vida, reivindican la autonomia del arte y los mundos posibles de la ficción literaria. Pero también, sus poéticas narrativas vistas desde otro ángulo, configuran dos aristas importantes para la renovación narrativa contemporánea. "Superstición" genealógica que surge tanto del deliberado socavamiento y deconstrucción del régimen

„1 Cfr. W. Theodor Adorno (1980) y BENJAMIN, Walter (1987, p.15-57 y 1991. p. 115-134).

12 Cfr. ALTAMIRO, Carlos y SARLO. Beatriz (1983, p. 127-17I) y SARLO. Beatriz. (1988. p. 95-120) 
clásico de la novela y de los protocolos del realismo decimonónico, en el caso de Macedonio Fernández, como de la revaluación y el trabajo de experimentación con los géneros. considerados hasta ese momento sobre los márgenes de la literatura. en el caso de Borges. Basta leer Museo de la Novela de la Elerna o Adriana Buenos Aires (Ulima Novela Mala) o los relatos de Borges para reconocer la ruptura con el canon hegemónico o el impulso de renovación narrativa que surgen de estos textos. ${ }^{13}$ Creo que a partir de esta " tradición", pueden leerse los mejores textos de Saer, Puig o Piglia para citar algunos nombres propios que son revaluados y tomados como puntos de referencia de una de las lineas narrativas de la llamada " joven" narrativa de los ' 90 '.

* Mi ideologia es contar una buena historia de la mejor manera posible"

(Rodrigo Fresan)

"Al referir una verdad que trasciende y abarca la realidad más inmediata. el narrador opta por renunciar. por inconsistente, al papel más bien módico de contador que lo condena la novela realista"

(Sergio Chefjec).

Haciendo una reducción de la multiplicidad de escrituras emergentes en los últimos '90' en la Argentina, los jóvenes escritores, se hallan asociados a dos grupos básicos que mantienen diferentes formas de relación con la tradición literaria, los medios masivos de comunicación y el mercado. $Y$ a su vez, configuran poéticas en enfrentamiento y disputa. Neotradicionalistas o estéticamente conservadores, experimentalistas o neovanguardistas. Los primeros, Rodrigo Fresan, Juan Forn, Guillermo Saccomanno, entre otros, ante la sospecha del agotamiento de la "experimentación" narrativa visible en ciertos textos de los ' 80 ', cultivan un retorno a la narración en los términos más clásicos y parecen profesar, la ilusión de una vuelta a los modos narrativos populares y decimonónicos -al estilo Dickens, Dumas o Balzac- cuando la televisión o los medios masivos y electrónicos no competían el espacio social de la literatura. Un deseo de recuperar lo narrativo a través de una trama bien construida o a partir de la

13 Cfr. BORGils. Jorge Luis. (1980) y Macedonio Fernández (1975 y 1988). 
"redondez" del relato clássico. Textos que intentan reelaborar un pacto de mimesis con el lector, con los deseos imaginarios del público televisivo (ver por ejemplo, de Rodrigo Fresán. Historia Argentina, 1991 o Vida de Santos, 1993). De alli que son relatos que dan importancia a la fábula y a la peripecie narrativa, con una temporalidad puesta al servicio del conflicto narrativo. Textos sin fragmentación y con escasa variación del sistema de enunciación o del punto de vista y que elaboran personajes en muchos casos, estereotipados o adscriptos al imaginario de la tipificación social, acercando de esta manera, los modos estéticos a los conflictos cotidianos (ver de Guillermo Saccomanno. Bajo Bandera, 1991 o Animales Domesticos, 1993). Son en su mayoria relatos unidireccionales, sin desvíos y con escasa ambigüedad que apuestan a las certidumbres del lector.

Sergio Chefjec y el grupo de los llamados experimentalístas (Daniel Guebel, Alan Pauls, Martín Caparrós, Carlos Eduardo Feiling) se apartan de los moldes clásicos de la narración y hacen del trabajo con la lengua, la problematización de la narración y de lo " real" (cfr. Chefjec. El Aire, 1992), la disolución de la temporalidad lineal y de los ensayos o pruebas a partir de los géneros "menores", hoy canonizados y dominantes tanto en el cine como en la literaturaobviamente me refiero al policial o trhiller, el relato de viaje o de aventuras y en menor medida el relato epistolar- (cfr. a modo de ejemplo Pauls, El Pudor del Pornografo, 1984 o del mismo autor El Coloquio, 1990; también de Feiling, El Agua Electrizada, 1992 o Un Poeta Nacional, 1993 o de Caparrós, La Noche Anterior, 1990, etc), o del uso estratégico de la fábula oriental o de lo exótico como marca posicional de distanciamiento, sus principales políticas de escritura (cfr. por ejemplo Guebel, La Perla del Emperador, 1990). Además, en muchos casos, se advierte una clara intención de cruzar las formas provenientes de la cultura popular o de masas con problemáticas propias de la alta cultura o de la vanguardia histórica. En este sentido, los juegos y descentramientos a nivel de la enunciación que desacralizan al narrador hegemónico de la novela decimonónica, el montaje de citas y la incorporación del discurso ajeno, la autorreflexividad e infiltración teórica, los saltos espacio-temporales, la ruptura con el pacto de mímesis del realismo y con el régimen del relato clásico, con el orden y la lógica causal de la novela decimonónica, son algunas de las estrategias narrativas de distanciamiento. 
"Quando si parla di $<<$ tradizione letteraria $\gg$ o di $<<$ successione $\gg$, ci si figura solitamente una linea retta che unisce il rappresentante più giovanne di un determinato ramo letterario al più anziano. $\mathrm{Ma}$ in realta la cosa e molto più complessa. Non si tratta di una linea retta continua. ma piuttosto della partenza. dell' allontanamento da un determinato punto: si tratta di una lotta “.(Jurij Tinianov)

¿ En qué contexto leerlos?. Habría por lo menos dos modos de leer el corpus de lo " nuevo". En el contexto de la tradición nacional, es decir, a partir del relato familiar que cada escritor hace de la serie nacional. Las polémicas que entablan con los horizontales y las relaciones de filiación, las apropiaciones y entrecruzamientos con las escrituras previas. $Y$ en el contexto de la traducción, las lecturas de novedades, adaptaciones y plagios de la serie extranjera. Usos y estrategias que remiten en un caso, a los modelos narrativos de Osvaldo Soriano y Enrique Fogwill; en el otro, a Lamborghini, Puig, Saer, Piglia o Aira. Pero también, reenvían a los marcos de referencia extranjeros. La persistencia del modelo de Thómas Bernhard en Alan Pauls y Sergio Chefjec, en la morosidad del relato y en el martilleo que marca la progresión de las palabras y los sucesos. John Cheever, el minimalismo norteamericano, representado entre otros por Raymond Curver y Tobias Wolff, la nueva narrativa anglosajona de los ' 80 ' con autores como Martín Amis, Salma Rushdie, Julian Barnes y John Irving y la narrativa " neodickensiana", sirven de base para la atención puesta en las costuras de la trama, la construcción de una suerte de epopeya de lo cotidiano y el retorno del influjo del exotismo oriental como marca diferenciadora. Estos últimos rastros pueden encontrarse en las narraciones de Rodrigo Fresán, Juan Forn, Sergio Bizzio o Daniel Guebel.

Creo que estas son algunas líneas de reflexión sobre la llamada joven narrativa argentina.

\section{RESUMEN}

El presente trabajo, intenta establecer un primer mapa de lectura sobre la producción narrativa de escritores argentinos que hoy rondan entre los 30 y 45 años. Articulado en base a la categoría de lo "nuevo". recorre el problema a partir de algunas 
aristas relevantes: la configuración del mercado de bienes simbólicos. las poéticas de la narración dominantes hacia los últimos '90' y su relación con cl contexto de la tradición nacional y la serie literaria extranjera.

Palabras-chaves: Poéticas-Mercado-Tradición.

\section{REFERENCIAS BIBLIOGRAFICAS}

\section{A) TEXTOS NARRATIVOS}

BORGES, Jorge Luis. Prosa Completa (Vol. 1 y II). Barcelona: Bruguera, 1980.

BIZZIO, Sergio. Infierno Albino. Buenos Aires: Sudamericana. 1991.

CAPARRÓS, Martín. Ansay o los Infortunios de La Gloria. Buenos Aires: Ada Korn Editora. 1984.

La Noche Anterior. Buenos Aires: Sudamericana, 1990.

El Tercer Cuerpo. Buenos Aires: Punto Sur. 1990.

CHEFJEC. Sergio. Moral. Buenos Aires: Punto Sur. 1990.

El Aire. Buenos Aires: Alfaguara Literaturas, 1992.

FEILING. Carlos Eduardo. El Agua Electrizada. Buenos Aires: Sudamericana. 1992. Un Poeta Nacional. Buenos Aires: Sudamericana. 1993.

FERNÁNDEZ. Macedonio. Museo De La Novela De La Eterna (Primera Novela Buena). Buenos Aires: Corregidor. 1975.

Adriana Buenos Aires (Ultima Novela Mala). Buenos Aires: Corregidor. 1988.

FORN, Juan. Nadar de Noche. Buenos Aires: Planeta . 1991.

FRESÁN. Rodrigo. Historia Argentina. Buenos Aires: Planeta . 1991.

I'ida de Santos. Buenos Aires: Planeta, 1993.

GUEBEL. Daniel. La Perla del Emperador. Buenos Aires: Emecé. 1990.

Los Elementales. Rosario: Beatriz. Viterbo. 1992.

LANATA. Jorge. Polaroids. Buenos Aires: Planeta, 1992.

PAULS, Alan. El Pudor del Pornografo. Buenos Aires: Sudamericana. 1984.

El Coloquio. Buenos Aires: Emecé. 1990.

GUILLERMO Saccomanno. Bajo Bandera. Buenos Aires: Planeta. 1991. Animales Domesticos. Buenos Aires: Planeta, 1994.

\section{B) ESTUDIOS}

ADORNO, Theodor Wiesenground. Teoria Estetica. Buenos Aires: Orbis, 1980.

ALTAMIRO. Carlos y SARLO, Beatriz: "Vanguardia y criollismo: la aventura de Martín Fierro". en: Ensayos Argentinos. de Sarmiento A La l'anguardiA. Buenos Aires:CEAL, p. 127-171. 1983. 
BARTHES, Roland: " La guerra de los lenguajes". en: El susurro del lenguaje. Mas alla de la Palabra Y la Escritura. Barcelona: Paidós Comunicación. 1987. p. 135-139. 1987.

BENJAMÍN. Walter: " La obra de arte en la época de su reproductibilidad técnica". en: Discursos ininterrumpidos I. Madrid: Taurus. p. 15-57. 1987.

: "El autor como productor" en Tentativas Sobre Brecht. Iluminaciones III Madrid: Taurus. p. 115-134, 1991.

BOURDIEU. Pierre: "Campo intelectual y proyecto creador". en: Problemas del Estructuralismo. México: Siglo XXI, 1971. p. 135-182, 1971.

BÜRGER. Peter. Teoria de la l'anguardia. Barcelona: Península, 1987. : "La declinación del modernismo" en Punto de l'ista. n"46: 40-48. 1993.

ROSA. Nicolás. Artefacto. Rosario: Beatriz Viterbo, 1992.

SAIDÓN, Grabiela (ed). Encuentro del Bosque. La Argentina como Escenario. Primera Reunion de Narradores Argentinos En el Hotel del Bosque de Pinamar. Buenos Aires: Sudamericana, 1993.

SARLO. Beatriz:" Vanguardia y Utopia", en: Una Modernidad Periferica: Buenos Aires 1920 Y 1930. Buenos Aires: Nueva Visión, p. 95-120, 1988.

:"El relativismo absoluto o cómo el mercado y la sociología rellexionan sobre estética", en: Punto de l'ista. $\mathrm{n}^{\circ}$ 48: 27-31. 1984.

SHÜCKING. Levin. El Gusto Literario. México: FCE. 1960. 